\title{
Brd4 links chromatin targeting to HPV transcriptional silencing
}

\author{
Shwu-Yuan Wu, ${ }^{1}$ A-Young Lee, ${ }^{1}$ Samuel Y. Hou, ${ }^{1}$ Jongsook Kim Kemper, ${ }^{2}$ \\ Hediye Erdjument-Bromage, ${ }^{3}$ Paul Tempst, ${ }^{3}$ and Cheng-Ming Chiang ${ }^{1,4}$ \\ ${ }^{1}$ Department of Biochemistry, Case Western Reserve University School of Medicine, Cleveland, Ohio 44106, USA; \\ ${ }^{2}$ Department of Molecular \& Integrative Physiology, University of Illinois, Urbana, Illinois 61801, USA; ${ }^{3}$ Molecular Biology \\ Program, Memorial-Sloan-Kettering Cancer Center, New York, New York 10021, USA
}

The E2 protein encoded by human papillomaviruses (HPVs) inhibits expression of the viral E6 oncoprotein, which, in turn, regulates p53 target gene transcription. To identify cellular proteins involved in E2-mediated transcriptional repression, we isolated an E2 complex from human cells conditionally expressing HPV-11 E2. Surprisingly, the double bromodomain-containing protein Brd4, which is implicated in cell cycle control and viral genome segregation, was found associated with E2 and conferred on E2 the ability to inhibit AP-1-dependent HPV chromatin transcription in an E2-binding site-specific manner as illustrated by in vitro reconstituted chromatin transcription experiments. Knockdown of Brd4 in human cells alleviates E2-mediated repression of HPV transcription. The E2-interacting domain at the extreme $\mathrm{C}$ terminus and the chromatin targeting activity of a bromodomain-containing region are both essential for the corepressor activity of Brd4. Interestingly, E2-Brd4 blocks the recruitment of TFIID and RNA polymerase II to the HPV E6 promoter region without inhibiting acetylation of nucleosomal histones $\mathrm{H} 3$ and $\mathrm{H} 4$, indicating an acetylation-dependent role of Brd4 in the recruitment of E2 for transcriptional silencing of HPV gene activity. Our finding that Brd4 is a component of the virus-assembled transcriptional silencing complex uncovers a novel function of Brd4 as a cellular cofactor modulating viral gene expression.

[Keywords: HPV; E2; AP-1; Brd4; chromatin transcription; gene silencing]

Supplemental material is available at http://www.genesdev.org.

Received May 9, 2006; revised version accepted June 28, 2006.

Bromodomains are acetyl-lysine targeting modules of $\sim 110$ amino acids commonly found in protein subunits of chromatin-modifying complexes (Winston and Allis 1999). These complexes work by covalently modifying histone tails or by using the energy of ATP to alter nucleosome structure. In addition to these chromatinmodifying enzymes, some transcriptional components, such as the TAF1 subunit of TFIID, also contain bromodomains able to recognize acetylated core histones at specific lysine residues (Kanno et al. 2004). The two tandem bromodomains found in TAF1 can bind simultaneously to two acetyl-lysine residues separated by seven or eight amino acids, such as acetylated K5/K12 and K8/ K16 of histone H4 (Jacobson et al. 2000). Other proteins with two tandem bromodomains, followed by an additional extraterminal (ET) domain, constitute the BET subfamily, which includes yeast Bdf1 and Bdf2, Drosophila Fs(1)h, and mammalian Brd2/Ring3/Fsrg1, Brd3/ Orfx/Fsrg2, Brd4/MCAP, and Brd5/Brdt (Denis et al. 2000; Dey et al. 2000; Matangkasombut et al. 2000; Houzelstein et al. 2002; and references therein). A unique feature of the BET family members lies in their ability to

${ }^{4}$ Corresponding author.

E-MAIL cmc23@cwru.edu; FAX (216) 368-3419.

Article published online ahead of print. Article and publication date are online at http://www.genesdev.org/cgi/doi/10.1101/gad.1448206. bind mitotic chromosomes. The chromatin targeting activity of bromodomain-containing proteins allows them to access chromatin and regulate gene activity through cell cycle progression.

In many virus-infected cells, the viral genome is similarly packaged into chromatin (Favre et al. 1977). Thus, the necessity for chromatin targeting is also critical for viral gene expression. In viruses, regulation of gene transcription normally relies on the presence of a virus-encoded master regulator, which is often a multifunctional protein capable of working with distinct sets of cellular factors to fine-tune gene activity. A well-known member is the human papillomavirus (HPV)-encoded E2 protein. The full-length E2 protein is a sequence-specific DNAbinding protein implicated in the control of viral DNA replication (Stenlund 2003; Abbate et al. 2004), transcription (Hou et al. 2002), cell cycle progression (Hwang et al. 1993), apoptosis (Blachon et al. 2005), senescence (Goodwin and DiMaio 2001), and viral genome maintenance and segregation (Abroi et al. 2004; Botchan 2004; McBride et al. 2004; Van Tine et al. 2004; You et al. 2004). Like many cellular transcription factors with different functional modules, E2 contains a C-terminal DNA-binding domain linked to the N-terminal transactivation domain by a flexible hinge. This configuration allows E2 to recognize distinct E2-binding sites 
$\left(\mathrm{ACCN}_{6} \mathrm{GGT}\right)$ with variable internal sequences (Hegde 2002). Although HPV E2, indeed, possesses weak transactivating activity (Hou et al. 2000), it normally functions as a transcriptional repressor in its natural host to inhibit the expression of viral E6 and E7 oncoproteins (Bernard et al. 1989). These virus-encoded oncoproteins, especially from high-risk genital HPVs that induce cervical cancers, are able to functionally inactivate p53 and $\mathrm{pRb}$ tumor suppressor proteins generally by targeting these cellular proteins for degradation (Münger et al. 2004). Recently, we have uncovered an additional mechanism for E6 inactivation of promoter-bound p53 function by inhibiting p300-mediated acetylation of p53 and nucleosomal core histones, independently of inducing p53 degradation (Thomas and Chiang 2005). This degradation-independent pathway is commonly employed by E6 encoded by both low-risk genital HPVs that are associated with benign genital warts and high-risk cancer-inducing HPVs.

Other than the full-length E2 protein, some types of HPVs also encode E2 variants that have the same Cterminal DNA-binding domain but without the N-terminal transactivation region (Chiang et al. 1991, 1992; Stubenrauch et al. 2000; Jeckel et al. 2003). These E2 variants, including the double-spliced E2C (dsE2C) protein encoded by low-risk HPV type 11 (HPV-11), typically act as transcriptional repressors that inhibit HPV E6 promoter activity via specific E2-binding sites (Hou et al. 2000). Surprisingly, while both full-length E2 (hereon referred to as E2) and dsE2C are able to suppress E6 promoter activity with HPV DNA templates in a cell-free transcription system reconstituted with recombinant TFIIB, TFIIE, TFIIF, and epitope-tagged RNA polymerase II (pol II), TFIID, and TFIIH (Hou et al. 2000), inhibition of HPV transcription in cultured cells appears to additionally require the $\mathrm{N}$-terminal region of $\mathrm{E} 2$ (Dowhanick et al. 1995; Demeret et al. 1997; Goodwin et al. 1998). Presumably the N-terminal domain is necessary for E2 association with undefined cellular proteins to inhibit E6 promoter activity from HPV chromatin that is assembled in the cell.

To identify cellular proteins involved in E2-mediated regulation of HPV chromatin transcription, we established several human cell lines that conditionally express HPV-11 E2 and dsE2C, respectively. Intriguingly, E2 was found to exist in two distinct complexes. Here, we describe the functional characterization of an E2 transcriptional silencing complex that contains E2 and the double bromodomain protein Brd4. Recombinant E2 and Brd4 are both necessary and sufficient for suppressing activator protein 1 (AP-1)-dependent HPV chromatin transcription in an E2-binding site-specific manner. An essential role for Brd4 as a transcriptional corepressor for E2-mediated inhibition of E6 promoter activity is further illustrated by reporter gene assays performed in transfected and stable Brd4-knockdown cells, and by RT-PCR and chromatin immunoprecipitation (ChIP) assays conducted in E2-expressing cells. Our results demonstrate that Brd4 recruits E2 for silencing of HPV chromatin transcription through the $\mathrm{N}$-terminal region of E2. This finding thus identifies Brd4 as the long-sought cellular cofactor involved in E2-mediated repression of HPV chromatin transcription and gene silencing of HPV E6 oncoprotein that antagonizes p53 tumor suppressor function.

\section{Results}

Brd4 is present in a cellular complex assembled by HPV-11 E2, but not by dsE2C

To identify cellular proteins mediating the nuclear activity of E2 and to bypass potential cytotoxicity triggered by constitutive $\mathrm{E} 2$ production in mammalian cells, we established several tetracycline-regulated E2 cell lines conditionally expressing either dual hexahistidine/FLAGtagged HPV-11 E2 (367 residues) or dual hexahistidine/ FLAG-tagged dsE2C that contains 11 amino acids derived from the E1 ORF linked to the C-terminal 149 residues of E2 (Hou et al. 2000). These proteins were efficiently induced, following the removal of tetracycline from cultured medium, in human embryonic kidney-derived 293 cells (Fig. 1A) and in human cervical carcinoma-derived HeLa cells (data not shown). The resulting cell lines were named 293-E2, 293-dsE2C, HeLa-E2, and HeLa-dsE2C, respectively.

To purify cellular proteins associated with the N-terminal domain of E2, we first fractionated 293-E2 nuclear extracts by $\mathrm{P} 11$ phosphocellulose ion-exchange chromatography. The majority of E2 was found in the P11 0.5 M $\mathrm{KCl}$ (P.5) fraction with some also detected in the $0.85 \mathrm{M}$ $\mathrm{KCl}$ (P.85) fraction (Fig. 1B, 293-E2). The E2 complex was then isolated from the P.5 fraction, designated as E2-P.5, by anti-FLAG immunoaffinity purification (Fig. 1B). Control purification was conducted in parallel from P.5 fractions prepared from both parental and dsE2C-expressing 293 cell lines. As described for E2, dsE2C was also found in both P.5 and P.85 fractions, although a greater part of dsE2C resided in the P.85 fraction (Fig. 1B, 293-dsE2C). When the purified E2-P.5 complex was separated by SDS-PAGE and visualized by silver staining, a major polypeptide of $\sim 200 \mathrm{kDa}$ and a protein doublet migrating at $\sim 120 \mathrm{kDa}$ were associated specifically with E2 (Fig. 1C, lanes 1-3). The 200-kDa band was identified as Brd4 and the $120-\mathrm{kDa}$ protein doublet was characterized to be SMC5 and SMC6 by mass spectrometry (Supplementary Figs. 1-3). The identity of Brd4 was further verified by Western blotting with an anti-Brd4 antibody (Fig. 1C, lanes 4-7). These results not only identified Brd4 as a cellular protein naturally associated with HPV E2 in the cell but also demonstrate that the association of Brd4 with E2 indeed requires the $\mathrm{N}$-terminal domain of E2, as Brd4 was not detected in the P.5 complex isolated from dsE2C-expressing cells.

\section{E2-P.5 is a transcriptional silencing complex capable of inhibiting AP-1-dependent HPV chromatin transcription}

Since Brd4 has two bromodomains able to bind acetylated chromatin (Dey et al. 2003), we wondered whether 
A

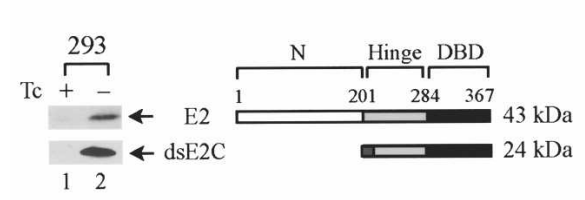

B

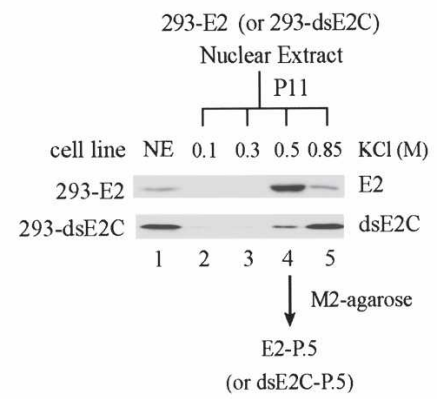

C

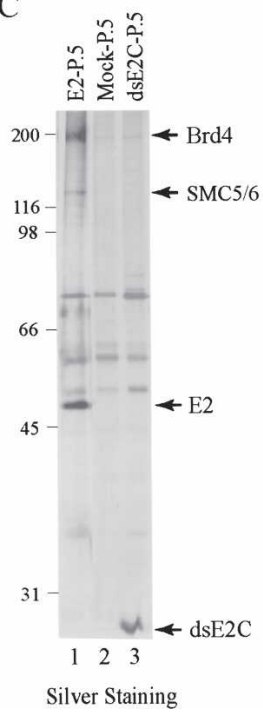

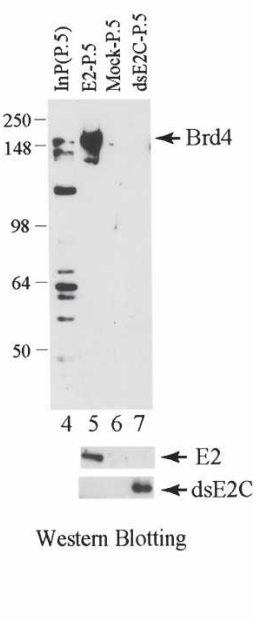

Figure 1. Identification of Brd4 as a component of a cellular complex assembled by HPV-11 E2, but not by dsE2C. (A) Inducible expression of hexahistidine/FLAGtagged E2 and dsE2C in 293-derived cell lines. Western blotting was performed with anti-hexahistidine antibodies using cell lysates, harvested in the presence $(+)$ or absence $(-)$ of tetracycline (Tc). (B) Purification scheme for E2-P.5 and dsE2CP.5 complexes. Detection of hexahistidine/FLAG-tagged E2 and dsE2C in nuclear extract (NE) and different P11 fractions, prepared from 293-E2 and 293dsE2C cell lines, respectively, was conducted by Western blotting with antiFLAG M2 monoclonal antibody. $(C)$ Brd4 is a component of the E2-P.5 complex. The presence of Brd4 in E2-P.5, dsE2CP.5, and a control sample purified in parallel from parental 293 cells (Mock-P.5) was analyzed by silver staining (lanes 1-3) and by Westernblotting with anti-mouse Brd4 antibodies (lanes 4-7, top panel). The existence of tagged E2 and dsE2C in the purified complexes was confirmed by Western blotting with anti-hexahistidine antibodies (lanes 5-7, bottom two rows). The P.5 fraction derived from 293-E2 cells was loaded as input (InP) control.

Brd4 might play a role in E2-mediated regulation of HPV E6 promoter activity. To explore this possibility, we first established an HPV chromatin transcription system using the p7072-70GLess $/ \mathrm{I}^{+}$template that contains the entire $1 \mathrm{~kb}$ of the HPV-11 upstream regulatory region (URR) with two well-characterized AP-1 recognition sequences and four E2-binding sites linked to a 377-basepair (bp) G-less cassette (Fig. 2A). We have previously shown that transcription in vitro from this HPV-11 URR-driven DNA template initiates at the correct start site and faithfully recapitulates E2-mediated regulation of E6 promoter activity as observed in vivo (Hou et al. 2000). This HPV-11 DNA template was assembled into chromatin by incubating with purified HeLa core histones, Drosophila nucleosome assembly factor ACF (a protein dimer reconstituted from recombinant Acf1 and FLAG-tagged ISWI), and recombinant human NAP-1 histone chaperone (Fig. 2B, lanes 1-3), according to the scheme (Fig. 2C) described previously for the assembly of a p53-dependent chromatin template (Thomas and Chiang 2005). Micrococcal nuclease (MNase) digestion was performed to verify formation of regularly spaced nucleosomes on the in vitro reconstituted HPV chromatin (Fig. 2D). To examine whether nucleosomes were also positioned at specific locations on HPV-11 chromatin similar to that of HPV-16 chromatin assembled with crude Drosophila S190 extracts (Stünkel and Bernard 1999|, we mapped the upstream boundary of a promoterproximal nucleosome by conducting primer extension on DNA fragments isolated from MNase-digested nucleosomes containing $>90 \%$ mononucleosomes and some residual dinucleosomes (see Supplementary Fig. 4). Indeed, the upstream boundary was mapped within the Sp1-binding site to nucleotide 33 of the HPV-11 genome (Fig. 2E), suggesting that nucleosomes are not only regu- larly spaced but also properly positioned (see below) on the in vitro reconstituted HPV-11 chromatin.

To ensure that the nucleosome positioning observed in vitro truly reflects the chromatin setting in vivo, we took advantage of the fact that HeLa cells harbor integrated HPV type 18 (HPV-18) genomes and thus provide a unique system for defining the in vivo boundaries of the E6 promoter-proximal nucleosome. Ligation-mediated PCR (LM-PCR), used for high-resolution mapping of nucleosomal structure in vivo (Kemper et al. 2004), was conducted with primer A and primer B that anneal to the HPV-18 E6 promoter region for pinpointing, respectively, the upstream and downstream boundaries of the promoter-proximal nucleosome (Fig. 2F). Indeed, a phased nucleosome was mapped precisely between HPV18 nucleotides 33 and 187 in the presence of T4 kinase, which enables the LM-PCR reactions (Fig. 2F, lanes 1-4). It is noted that a distinct band detected at nucleotide 7816 between the AP-1 and \#2 E2-binding sites likely represents the downstream boundary of an adjacent upstream nucleosome with several fainter bands presumably caused by incomplete MNase digestion of the linker DNA (Fig. 2F, lanes 1,5). An extra band with size slightly smaller than that of the boundary-demarking band (Fig. 2F, lane 3) may be a sign of nucleosome breathing (Mellor 2005) typically occurring at the DNA entry and exit sites preferentially cleaved by MNase. Remarkably, when in vitro reconstituted HPV-18 chromatin containing the whole viral genome was similarly analyzed by primer extension, we found that the upstream boundary of the HPV-18 promoter-proximal nucleosome was mapped to the identical position as identified in HeLa cells (Fig. 2F, cf. lanes 5 and 6). The size difference between the in vivo (126 nucleotides [nt]) and in vitro (103 $\mathrm{nt})$ bands is due to the length of the ligation linker (23 nt) 
Wu et al.
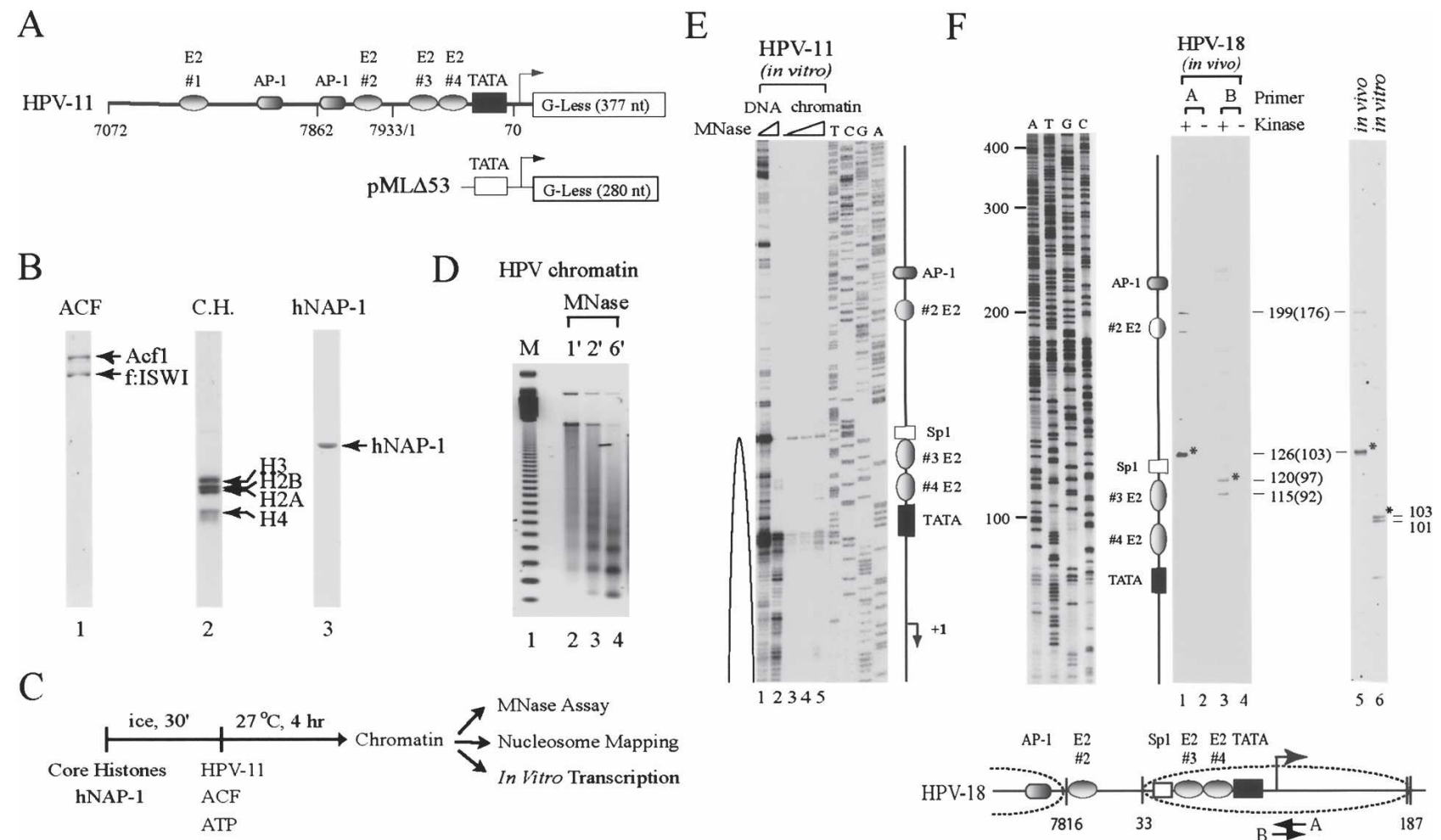

12345

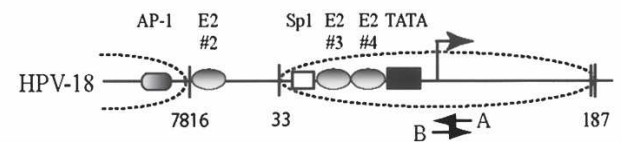

Figure 2. Mapping of promoter-proximal nucleosome positioning on the HPV E6 promoter in vitro and in vivo. $(A)$ G-less cassette templates used for chromatin assembly and transcriptional analysis. (B) Purified ACF, core histones (C.H.), and hNAP-1 used for chromatin assembly. $(C)$ Outline of in vitro chromatin assembly for MNase digestion, nucleosome mapping, and transcriptional analysis. $(D)$ Formation of regularly spaced nucleosomes on in vitro reconstituted HPV-11 chromatin analyzed by MNase digestion at different incubation times. A 123-bp DNA ladder was used as a size marker (M). (E) Mapping the upstream boundary of a promoterproximal nucleosome assembled on in vitro reconstituted HPV-11 chromatin. Increasing MNase digestion times were used to cleave HPV-11 chromatin or the same mock-assembled (i.e., protein-free) DNA. A size marker ( $\mathrm{T}, \mathrm{C}, \mathrm{G}$, and A lanes) was generated from a DNA sequencing reaction using the same primer for nucleosome mapping. Transcription factor-binding sites in the E6 promoterproximal region are indicated on the right, with +1 specifying the transcription start site and the oval shape (drawn on the left) depicting the nucleosome-embedded region. $(F)$ Nucleosome positioning on HPV-18 chromatin isolated from HeLa cells (in vivo) and on in vitro reconstituted HPV-18 chromatin. LM-PCR was conducted with primer A or primer B, in the presence $(+)$ or absence $(-)$ of T4 DNA kinase, for in vivo mapping of nucleosome boundaries, whereas primer extension (with primer A) was used for in vitro analysis. Numbers in parentheses represent the lengths of DNA fragments extending from the $5^{\prime}$ end of the primer to the downstream (lane 3) or upstream (lanes 1,5,6) boundary of the E6 promoter-proximal nucleosome (indicated by an asterisk). The number preceding each parenthesis additionally includes the length of the linker DNA (23 nt) used for LM-PCR. A DNA size marker, generated from a sequencing reaction, is shown on the left.

included for in vivo LM-PCR analysis. Thus, our in vitro reconstituted HPV chromatin, indeed, reflects the physiological chromatin environment. Moreover, it suggests that HPVs carry natural nucleosome positioning elements that facilitate formation of phased nucleosomes on the viral genome (Stünkel and Bernard 1999; this study), thereby underlying the formation of viral minichromosomes observed earlier in infected cells (Favre et al. 1977).

The successful reconstitution of HPV chromatin faithfully recapitulating in vivo nucleosome phasing allows us to address the role of cellular proteins modulating HPV transcription in vitro with well-defined components. Since transcription is generally suppressed by nucleosomes assembled on the promoter region and the initiation of transcription from the silenced chromatin typically requires an activator and a histone acetyltrans- ferase such as p300 (Thomas and Chiang 2005), we had screened several viral and cellular transcription factors for their ability to initiate transcription from HPV chromatin in conjunction with acetyl-CoA and p300 following the outlined protocol (Fig. 3A). Intriguingly, when purified p300 and AP-1 (reconstituted from hexahistidine-tagged human c-Fos and untagged human c-Jun) (Fig. 3B) were incubated together with HPV-11 chromatin and acetyl-CoA prior to the addition of HeLa nuclear extract, ribonucleoside triphosphates, and an internal DNA control template (pML $\Delta 53$ ) that contains only the adenovirus major late core promoter preceding a 280 -bp G-less cassette, we could detect significant transcription from silenced HPV chromatin (Fig. 3C, lanes 2 vs. 1,4). A small (approximately twofold) effect of AP-1- and acetyl$\mathrm{CoA} / \mathrm{p} 300$-dependent stimulation was also seen with HPV DNA template (Fig. 3C, lanes 1-4 vs. 5-8). Further 
A

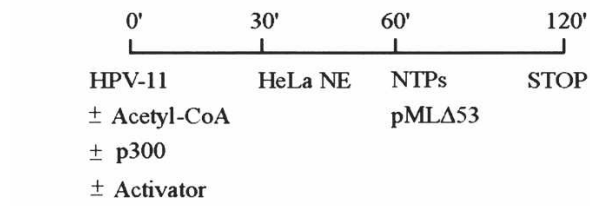

C

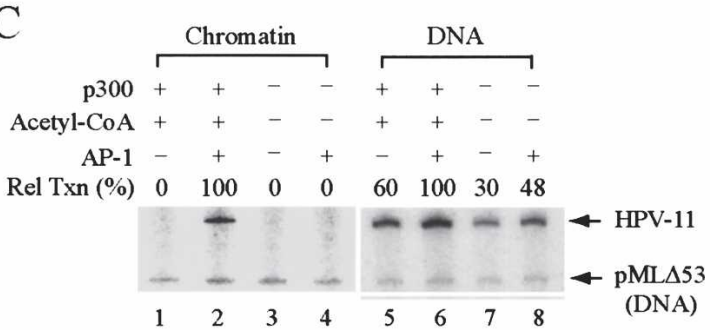

E

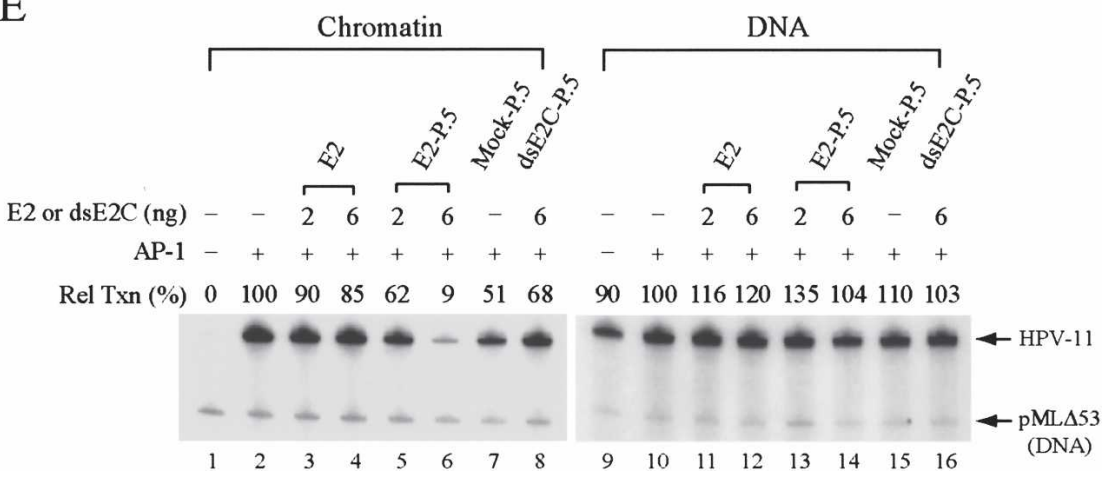

B

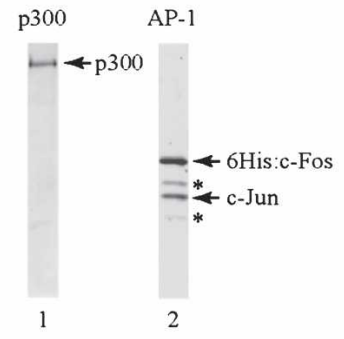

D

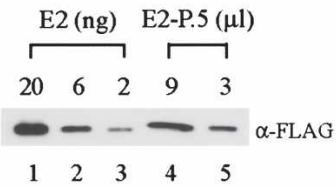

Figure 3. E2-P.5 inhibits AP-1-dependent HPV transcription specifically from chromatin, but not from DNA template. $(A)$ Outline of transcription assay with HPV-11 chromatin or DNA template. (B) Purified p300 and AP-1 used for transcription assays. Asterisk indicates degradation products derived from hexahistidine-tagged c-Fos. $(C)$ p300 and acetyl-CoA are critical for AP-1mediated activation from HPV chromatin. In vitro transcription was performed in HeLa nuclear extracts (NE) using HPV-11 chromatin or DNA template, in the presence $(+)$ or absence $(-)$ of $\mathrm{p} 300$, acetyl-CoA, and AP- 1 as indicated. The pML $\Delta 53$ control DNA template was added at the same time with nucleoside triphosphates (NTPs), as outlined in $A$. (D) Normalization of recombinant E2 and the E2-P.5 complex by Western blotting. (E) E2-P.5, but not recombinant E2 or dsE2C-P.5 and Mock-P.5, significantly represses transcription from HPV chromatin. In vitro transcription was performed as described in $C$ in the presence of p300 and acetyl-CoA, with (+) or without (-) AP-1, E2, E2-P.5, and dsE2C-P.5 added at the beginning of the reaction with HPV chromatin or DNA template. inclusion of an increasing amount of the E2-P.5 complex, normalized with recombinant E2 protein (Fig. 3D), suppressed HPV transcription specifically from chromatin (Fig. 3E, lanes 5,6 vs. 2, lanes 13,14 vs. 10). Only a modest effect was seen with recombinant E2, dsE2C-P.5, and control P.5 complexes (Fig. 3E, lanes 2-4,7,8). This finding suggests that the Brd4-containing E2-P.5 complex possesses transcriptional silencing activity specific to chromatin templates.

\section{E2-P.5 complex-mediated silencing of AP-1-dependent $H P V$ chromatin transcription requires promoter-proximal E2-binding sites}

To define whether transcriptional silencing mediated by the E2-P.5 complex requires E2 target sequences in the HPV URR, we used a set of truncated HPV-11 templates containing one AP-1-binding site and mutations in the \#2, \#3, and \#4 E2-binding sites, either individually or in combination (Fig. 4A), and assembled them, respectively, into chromatin. Regularly spaced nucleosomes were formed on each of these templates (Fig. 4B), similar to that observed with the DNA construct containing a longer URR (see Fig. 2D). Mutations of all three E2-binding sites (234M) almost completely alleviated repression by the E2-P.5 complex (Fig. 4C, lanes 5-8). Dissection of individual E2-binding sites indicated that the \#4, rather than \#2 or \#3, E2-binding site was most critical for repression by the E2-P.5 silencing complex (Fig. 4D, lanes 1-12), a conclusion further supported by the pairwise mutational studies (Fig. 4D, lanes 13-24) and also by previous in vivo and in vitro assays (Hou et al. 2000 and references therein).

Recombinant Brd4 and E2 are sufficient for E2-P.5 silencing activity

The abundance of Brd4 in the E2-P.5 complex, relative to the other minor bands such as SMC5 and SMC6, raised the possibility that Brd4 alone may be sufficient to confer on E2 the ability to silence HPV chromatin transcription. To test this hypothesis, we purified recombinant FLAG-tagged Brd4 (f:Brd4) protein, alone or together with recombinant HA-tagged E2 (HA:E2), from insect cells infected with respective recombinant baculoviruses (Fig. 5A). The existence of PEST sequences, frequently found in highly labile proteins, and the large size of recombinant Brd4 inevitably lead to some degradation species. Nevertheless, HA:E2 was copurified from coinfected insect cells with f:Brd4, which was pulled down by anti- 
Wu et al.

A

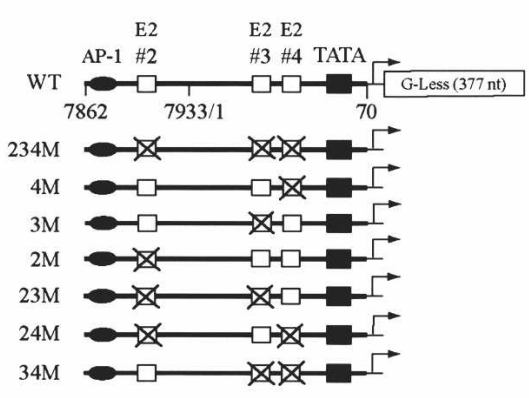

B

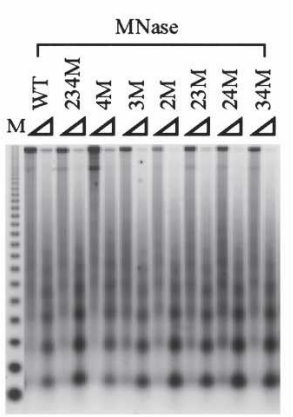

$\mathrm{C}$
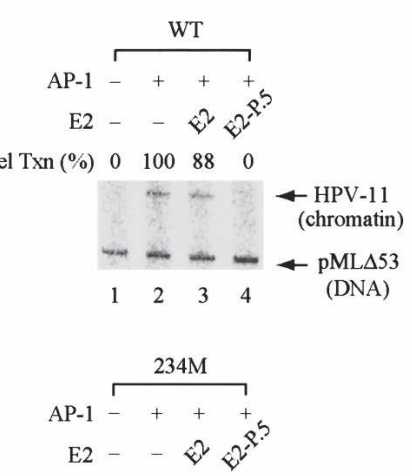

Rel Txn (\%) $0100 \quad 70 \quad 56$

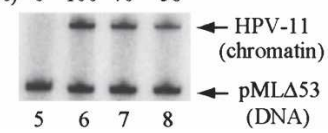

$\mathrm{D}$

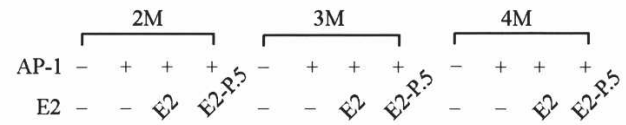

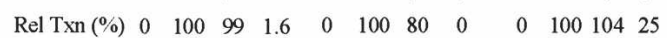
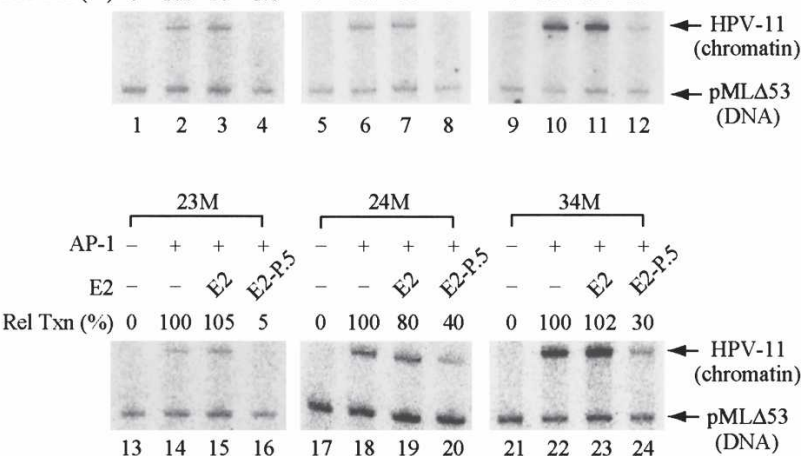

Figure 4. The \#4 promoter-proximal E2-binding site is critical for transcriptional silencing by the E2-P.5 complex. (A) HPV-11 G-less cassette templates used for transcriptional analysis. Protein-binding sites are indicated on the map with mutated E2-binding sites marked by X. $(B)$ Formation of regularly spaced nucleosomes on in vitro reconstituted HPV-11 chromatin templates analyzed by MNase digestion. A 123-bp DNA ladder was used as a size marker (M). $(C, D)$ The \#4 E2-binding site is critical for transcriptional silencing by the E2-P.5 complex. In vitro transcription was performed with HPV chromatin templates as indicated, in the presence of p300 and acetyl-CoA, with (+) or without (-) AP-1 and recombinant E2 or an equivalent amount of the E2-P.5 complex. Transcription from each chromatin template was repeated at least three times, and only a representative set of data from each panel is shown.

FLAG M2 monoclonal antibody-linked agarose beads (Fig. 5A, cf. lanes 2 and 3). No HA:E2 was isolated together with $\mathrm{C}$-terminally truncated f:Brd4 missing the E2-interacting domain (see below) during coinfection and complex purification (data not shown). These results suggest that E2 and Brd4, produced in baculoviruscoinfected insect cells, indeed form a preassembled complex. The availability of these recombinant proteins as individual entities or as a preassembled complex (Brd4/ E2) made it possible for us to examine directly their inhibitory effect on HPV chromatin transcription. As shown in Figure 5B, the presence of both Brd4 and E2 significantly suppressed AP-1-dependent transcription specifically from HPV chromatin but not from DNA templates (Fig. 5B, cf. lanes 5,6 and 1, and lanes 7-12). Brd4 alone appeared to slightly reduce transcription activity from both HPV chromatin and HPV DNA templates (Fig. 5B, lanes 4 vs. 2, lanes 10 vs. 8), likely due to squelching of some cellular factors involved in HPV transcription. Furthermore, inhibition of chromatin transcription by Brd4 and E2 seemed to be activator-specific, as the Brd4-E2 silencing complex failed to antagonize Gal4-
VP16-mediated transcription from a chromatin template $\left(\mathrm{pG}_{5} \mathrm{MLT}\right)$ containing five Gal4-binding sites (Fig. 5C).

The availability of recombinant Brd4 also allowed us to verify whether Brd4, indeed, interacts directly with the N-terminal domain of E2 (see Fig. 1C; You et al. 2004). Immobilized GST and GST fusions of HPV-11 E2, dsE2C, and an E2 mutant containing only the N-terminal amino acids 1-200 were incubated individually with recombinant $\mathrm{f}:$ Brd4. As shown in Figure 5D, f:Brd4 only associates with the full-length (FL) and the N-terminal 200 residues of E2, and does not interact with dsE2C. This result demonstrated that Brd4 indeed interacts directly with the $\mathrm{N}$-terminal domain of E2, consistent with the finding that Brd4 is present in the cellular complex assembled by E2, but not by dsE2C (see Fig. 1C). A reciprocal interaction assay was also carried out to map the E2-interacting domains on Brd4. Immobilized GSTE2(FL) or GST alone was incubated, respectively, with a series of bacterially expressed $\mathrm{f}: \mathrm{Brd} 4$ protein domains spanning the entire molecule (Fig. 5E, left and middle panels). The bound proteins were then detected by Western blotting with anti-FLAG M2 monoclonal antibody. 
A

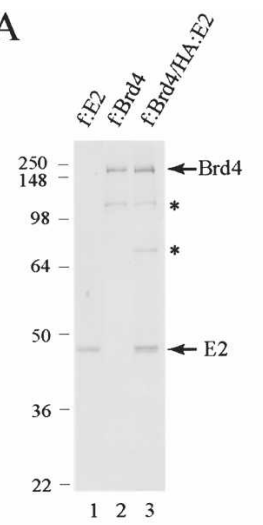

B
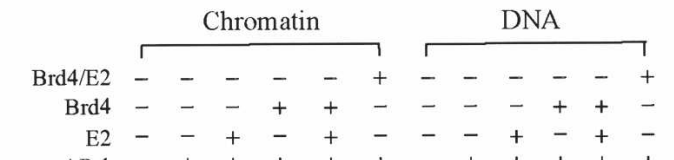

$\mathrm{E} 2--+-+--+$

$\begin{array}{lllllllllllll} & 40 & & \end{array}$

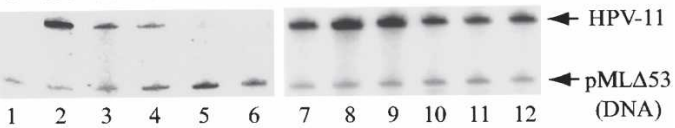

D
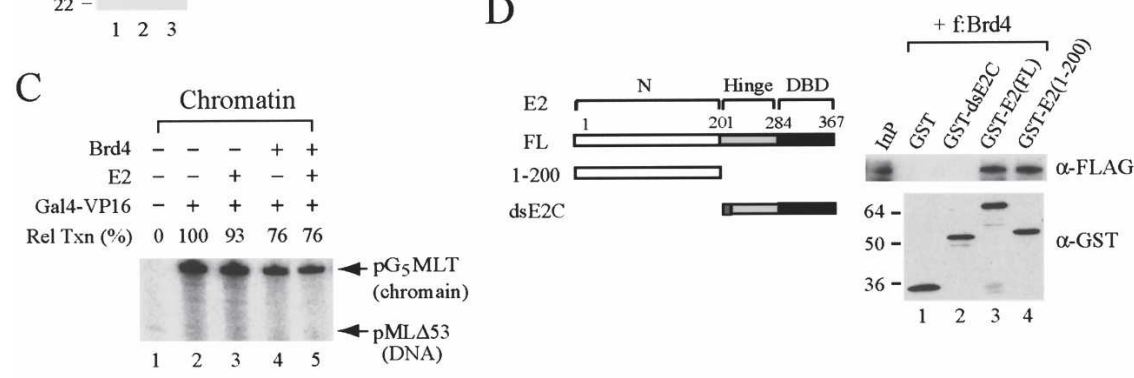

E

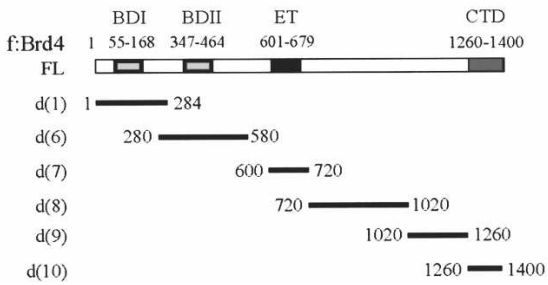

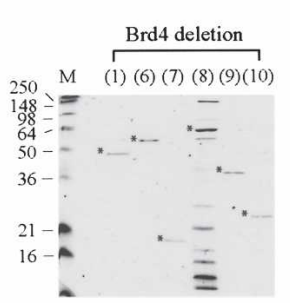

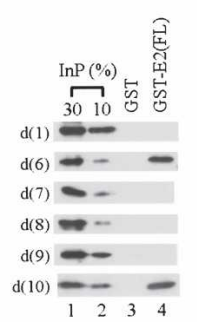

Figure 5. Recombinant $\mathrm{Brd} 4$ is necessary and sufficient for E2-mediated silencing of HPV chromatin transcription. (A) Purified recombinant Brd4 and the in vivo reconstituted recombinant Brd4-E2 complex. Proteins purified from insect Sf9 cells were resolved on a gel and visualized by Coomassie blue staining. Asterisk indicates degradation products derived from FLAG-tagged Brd4. $(B)$ Brd4 and $\mathrm{E} 2$, reconstituted in vivo (Brd4/E2) or individually combined in vitro, strongly inhibit HPV transcription from chromatin. In vitro transcription was performed as described in Figure 3E, except that recombinant Brd4 and recombinant E2 were used as indicated. (C) The E2-Brd4 complex does not inhibit Gal4-VP16-mediated chromatin transcription. In vitro transcription was performed as described in $B$, except that Gal4-VP16 and a chromatin template containing five Gal4binding sites ( $\left.\mathrm{pG}_{5} \mathrm{MLT}\right)$ were used in the experiment. $(D)$ The $\mathrm{N}$-terminal domain of E2 is necessary for interaction with Brd4. A GST pull-down assay was performed by incubating recombinant FLAG-tagged Brd4 (f:Brd4) with immobilized GST-E2(FL), GST-E2(1-200), or GST-dsE2C as indicated. Bound $\mathrm{f}: \mathrm{Brd} 4$ was then detected by Western blotting with anti-FLAG M2 monoclonal antibody. $(E)$ The extreme $\mathrm{C}$ terminus and a region containing the second bromodomain of Brd4 are involved in E2 interaction. A GST pull-down assay was per-

formed by incubating individually purified FLAG-tagged Brd4 deletion domains (middle panel, Coomassie-stained gel) with immobilized GST or GST-E2(FL). Bound f:Brd4 domains were then detected by Western blotting with anti-FLAG M2 monoclonal antibody. Amino acid residues spanning BDI and BDII, an ET domain, and the CTD of Brd4 are depicted on the left.

Clearly, two separate E2-interacting domains were identified on Brd4: one at the extreme C-terminal domain (CTD) and the other in a region containing the second bromodomain (Fig. 5E, right panel, lanes 3,4). Both regions of Brd4 are important for Brd4-mediated inhibition of HPV transcription (see below).

Knockdown of Brd4 in cultured human cells alleviates E2-mediated repression of E6 promoter activity

The identification of Brd4 as the cellular protein mediating E2 repression of HPV chromatin transcription in vitro suggests that Brd4 functions as a transcriptional corepressor for E2-mediated inhibition of HPV E6 promoter activity in vivo. To explore this possibility and to provide a functional link for Brd4 involvement in E6 promoter regulation, we conducted a reporter gene assay by transfecting pGL7072-161, which contains the HPV-11 URR-E6 promoter linked to a luciferase reporter, with different amounts of E2 and dsE2C expression plasmids, in the absence or presence of a Brd4 expression construct, into human cervical carcinoma-derived C-33A cells. Indeed, Brd4 significantly enhanced E2-mediated repression of E6 promoter activity in a dose-dependent manner, while it had no effect on dsE2C-mediated repression (Fig. 6A). In this transient transfection assay, dsE2C strongly inhibits E6 promoter activity due to its overexpression relative to E2 (Fig. 6A, lanes 1,2), whose expression in the crude lysate was below the detectable level unless a proteasome inhibitor, such as lactacystin or MG132, was included in the medium to prevent proteasome-mediated degradation of E2 through its N-terminal domain (Fig. 6A, lanes 3-5; see also Bellanger et al. 2001). The Brd4-independent repression exhibited by dsE2C likely works by inhibiting the assembly of preinitiation complexes on the nucleosome-free E6 promoter region or by squelching essential cellular factors modulating E6 promoter activity. These possibilities are in agreement with our previous finding that an equivalent amount of E2 and dsE2C can both suppress E6 promoter activity by preventing TFIIF-escorted pol II entry to the promoter region, as evidenced by in vitro transcription assays conducted with limiting amounts of general transcription components and with nucleosome-free DNA templates (Hou et al. 2000). Nevertheless, this transient transfection and reporter gene assay clearly verifies the 
Wu et al.

A
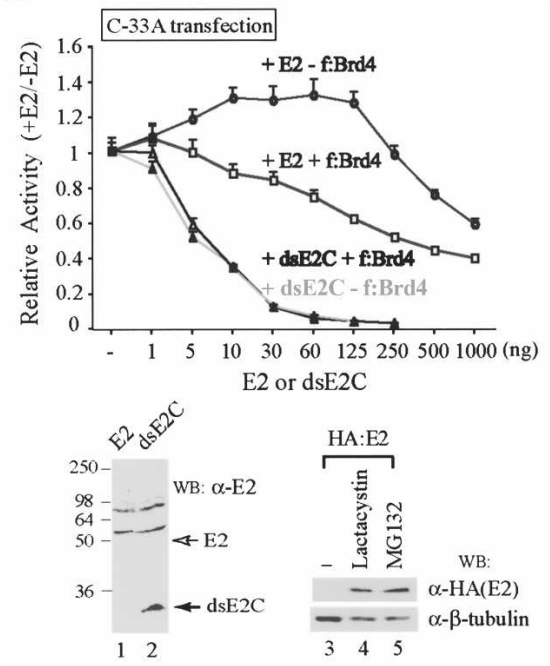

$\mathrm{D}$

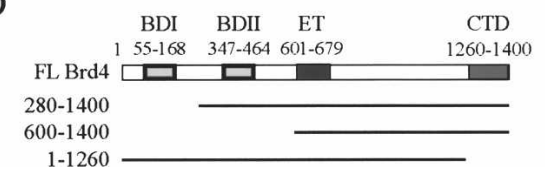

B

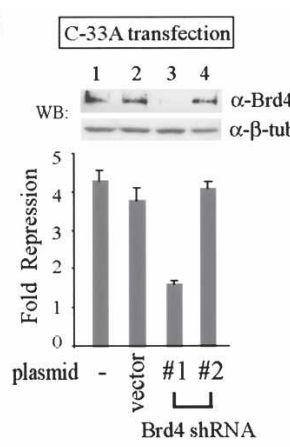

E

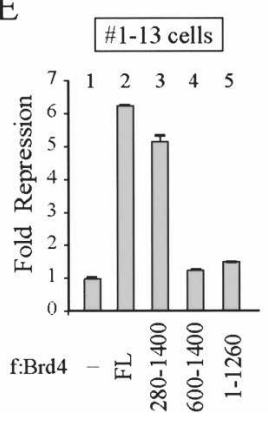

C
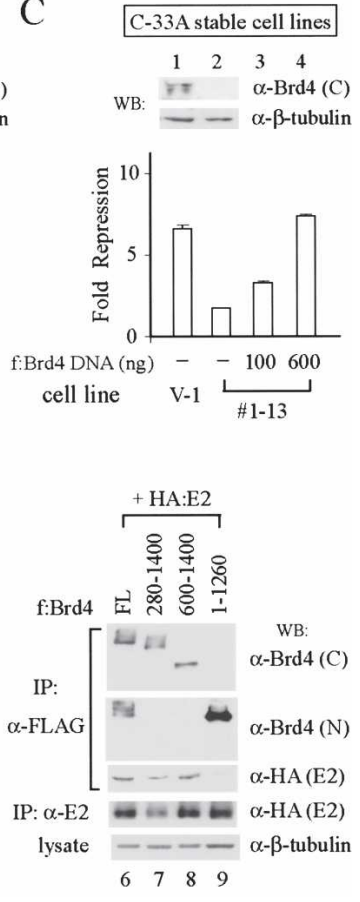

Figure 6. Knockdown of Brd4 significantly alleviates E2-mediated repression of HPV transcription in cultured human cells. $(A)$ Brd4 enhances repression of HPV E6 promoter activity mediated by E2, but not dsE2C, in transfected human C-33A cells. Transfection in C-33A cells was conducted by cotransfecting an HPV-11 URR-E6 promoter-driven reporter (pGL7072-161) with increasing amounts of an E2 (pRSE2-11) or dsE2C (pRS-CM4) expression plasmid, in the absence (-) or presence (+) of an f:Brd4 expression plasmid. Luciferase activity was measured $48 \mathrm{~h}$ after transfection. Relative activity represents the ratio of luciferase activity measured in the presence over the absence of E2. Detection of protein expression was performed as described in Materials and Methods. The open arrow points to the putative position of E2. (B) Knockdown of Brd4 alleviates E2-mediated repression in a transient transfection assay. C-33A cells were transfected with pGL7072-161, an HA-tagged HPV-11 E2 expression plasmid (pcDNA3-HA:11E2, 200 ng), and a retroviral vector expressing \#1 or \#2 Brd4 shRNA. Cell lysates were harvested $24 \mathrm{~h}$ later for Western blotting and luciferase analyses. (C) Restoration of E2-mediated repression of HPV-11 E6 promoter activity by transfecting a wild-type Brd4 expression plasmid into a C-33A-derived cell line constitutively expressing \#1 Brd4 shRNA. The pGL7072-161 reporter plasmid was cotransfected with pcDNA3-HA:11E2 (400 ng), in the absence (lanes 1,2) or presence (lanes 3,4) of an f:Brd4 expression plasmid into \#1-13 cells that express \#1 Brd4 shRNA or into vector-harboring V-1 cells. Western blotting and luciferase assays were then carried out as described in $B$. $(D)$ Schematic of the protein domains in mouse Brd4 proteins. $(E)$ The CTD and a bromodomain-containing region are both necessary for the corepressor activity of Brd4. Reporter gene assay was carried out in \#1-13 cells as described in C, except that different Brd4 deletion constructs were used for comparison with the full-length (FL) Brd4 clone. Detection of the expressed proteins by coimmunoprecipitation and Western blotting was conducted as described in Materials and Methods. Antibodies against the C-terminal region (residues 1199-1362) or the $\mathrm{N}$-terminal domain (residues 149-284) of human Brd4 were used for Western blotting.

functional importance of Brd4 interaction with the $\mathrm{N}$ terminal domain of E2.

To demonstrate that Brd4 is a bona fide transcriptional corepressor for E2 function in vivo, we evaluated the efficiency of E2-mediated repression of the E6 promoter by reducing the cellular level of Brd4 using a retroviral vector expressing small hairpin RNA (shRNA) specifically designed for Brd4 knockdown. Cotransfection of the \#1 Brd4 shRNA plasmid that produces small interfering RNA (siRNA) corresponding to nucleotides 228246 (i.e., the sequence coding for amino acids 76-82) of human Brd4 with pGL7072-161 and an E2-expressing plasmid significantly alleviated E2-mediated repression of E6 promoter activity due to a reduction of the Brd4 protein (Fig. 6B, lanes 1-3). The \#2 Brd4 shRNA plasmid that generates siRNA matching nucleotides 423-441, located further downstream from the \#1 sequence, was in- effective in diminishing E2 repressing activity, because the level of endogenous Brd4 was not reduced (Fig. 6B, lane 4). The success of using the \#1 shRNA plasmid for Brd4 knockdown in transient transfection prompted us to establish stable C-33A-derived cell lines constitutively expressing \#1 Brd4 shRNA. Indeed, repression of E6 promoter activity by E2 was dramatically reduced in stable Brd4-knockdown cells (\#1-13) relative to a control cell line (V-1) carrying only the vector, again in parallel with the drop of Brd4 in \#1-13 cells (Fig. 6C, lanes 1,2). Similar results were also obtained with other independent C-33A isolates expressing \#1 Brd4 shRNA /data not shown). It should be mentioned that knockdown of Brd4 did not change the level of E2 expression in both transiently transfected C-33A and its derived shRNA cell lines under various conditions tested, although the doubling time in stable knockdown cells has been extended 
from 24 to $48 \mathrm{~h}$ (data not shown). To confirm that a lack of efficient E2-mediated repression in \#1-13 cells was actually caused by the deficiency of Brd4 and to further exclude any indirect effect that might influence E6 promoter activity, we reintroduced Brd4 back to the cells by cotransfecting with the reporter and E2 plasmids an increasing amount of the $\mathrm{f}: \mathrm{Brd} 4$ expression plasmid. As expected, exogenous Brd4 efficiently restored E2-mediated repression of $\mathrm{E} 6$ promoter activity in a dose-dependent manner (Fig. 6C, lanes 3,4 vs. 1,2), demonstrating that the availability of Brd4, indeed, accounts for the efficiency of E2 repressing activity. We thus conclude that Brd4 is the cellular corepressor essential for E2-mediated repression of HPV E6 promoter activity.

We next used the stable Brd4-knockdown cells to define the functional role of the Brd4 CTD and the second bromodomain-containing region that are involved in E2 interactions (see Fig. 5E). Three truncated Brd4 mutants that selectively remove the first bromodomain (BDI), the second bromodomain (BDII), and the CTD (Fig. 6D) were transfected into \#1-13 cells, respectively, and compared with the full-length Brd4 for their ability to restore E2mediated repression of E6 promoter activity. Deletion of the BDI (construct 280-1400) slightly reduced the corepressor activity of Brd4 (Fig. 6E, lanes 2,3 vs. 1). Further removal of the BDII region (construct 600-1400) or deletion of the CTD (construct 1-1260) completely abolished the ability of Brd4 to support E2 repressing activity (Fig. $6 \mathrm{E}$, lanes 4,5 vs. 1). This functional assay demonstrates that the regions including the E2-interacting domains are also important for the corepressor function of Brd4. To ensure that FLAG-tagged full-length and truncated Brd4 proteins were all properly expressed in \#1-13 cells, we examined the levels of protein expression from these Brd4 expression plasmids by analyzing the anti-FLAG antibody pulled-down immunoprecipitates and detected the exogenously expressed Brd4 proteins with polyclonal antibodies that recognize either the C-terminal residues (1199-1362) or the N-terminal region (149-284) of human Brd4. While an approximately equivalent amount of full-length Brd4 and the 280-1400 and 600-1400 mutants was detected by the anti-Brd4 C-terminal antibodies, the 1-1260 mutant was detected by the anti-Brd4 N-terminal antibodies to a level significantly higher than that of the full-length protein detected by the same antibodies (Fig. 6E, lanes 6-9, top two panels). Even so, the 1-1260 mutant still failed to restore E2-mediated repression in the Brd4-knockdown cell (Fig. 6E, cf. lanes 2 and 5). Consistent with the in vitro pull-down assay, deletion of the CTD abolished Brd4 interaction with HPV E2 in the cotransfection assay (Fig. 6E, lanes 6,9, third row). Unexpectedly, the 600-1400 mutant still associated with E2 (Fig. 6E, lanes 6 vs. 8, third row), suggesting that the CTD is the predominant E2-interacting domain in the context of the full-length Brd4 protein and the failure of the BDI- and BDII-deleted 600-1400 mutant in re-establishing E2-mediated repression (Fig. 6E, lanes 4 vs. 1,2) is likely due to the inability of the doublebromodomain-deficient Brd4 mutant to bind chromatin (see below). The level of the E2 protein was not altered by coexpressed 600-1400 and 1-1260 mutants (Fig. 6E, lanes 8,9 vs. 6 , fourth row), thus excluding the possibility that the loss of E2-mediated repression was due to reduced E2 expression in these Brd4 mutant-expressing cells.

Brd4 enhances E2 recruitment to the E6 promoter region, resulting in reduced association of TFIID and pol II without altering the acetylation status of core histones

To examine whether the Brd4-E2 silencing complex inhibits E6 promoter activity by preventing the recruitment of the general transcription machinery, we carried out RT-PCR and ChIP assays with HeLa and HeLa-E2 cells that harbor endogenous HPV-18 genomes. Indeed, expression of E2 in HeLa-E2 cells significantly reduced the level of E6 transcripts (Fig. 7A). This result correlated with lower amounts of TAF1 and pol II recruitment to the endogenous E6 promoter (Fig. 7B, cf. lanes 6 and 7 in both panels), but not to the TAF7 gene (see Supplementary Fig. 5A). The ChIP assay, performed with a primer pair that amplifies a promoter-proximal DNA fragment containing \#3 and \#4 E2-binding sites and the TATA box of the E6 promoter (Fig. 7C), also revealed that Brd4 was already prebound to the E6 promoter in the absence of E2 and that the presence of E2 (detected by both anti-E2 and anti-hexahistidine antibodies) did not change the level of promoter-bound Brd4 (Fig. 7B, cf. lanes 5 in both panels). This finding suggests that E2 does not work by enhancing Brd4 corepressor recruitment to the promoter region. On the other hand, prebound Brd4 might play a critical role in facilitating E2 recruitment to the E6 promoter region. Surprisingly, the levels of acetylated and total histone $\mathrm{H} 3$ and $\mathrm{H} 4$ were not altered following the recruitment of E2 (Fig. 7B, lanes 8,9; see Supplementary Fig. $5 \mathrm{~B}$ ), indicating that Brd4 binding to acetylated core histones may be a prerequisite for efficient recruitment of E2 to the E6 promoter region. To explore this possibility, we performed ChIP assays following treatment of freshly thawed HeLa-E2 cells that express only limiting amounts of E2 with the histone deacetylase inhibitor sodium butyrate to enhance the level of acetylated chromatin in the cell and then examined the recruitment of Brd4 and E2 to the E6 promoter region as well as to the \#1 E2-binding site at $-0.5 \mathrm{~kb}$ and to the $\mathrm{L} 1$ region at $-1 \mathrm{~kb}$ upstream of the transcription start site (Fig. 7C). Under the condition in which a low concentration of E2 fails to bind stably to the endogenous HPV-18 genome, treatment of sodium butyrate, indeed, enhanced the levels of acetylated but not total histone $\mathrm{H} 4$ at the promoterproximal region and also at -0.5 - and $-1-\mathrm{kb}$ regions (Fig. 7D, lanes 5 vs. 10; see Supplementary Fig. 5C) without altering the level of E2 expression (data not shown). Accordingly, binding of E2 to both the promoter-proximal and $-0.5-\mathrm{kb}$ regions was significantly enhanced by the improved association of Brd4 with acetylated chromatin (Fig. 7D, lanes 3-5 vs. 8-10, top two rows). In contrast, no enhanced recruitment of Brd4 was observed at the $-1-\mathrm{kb}$ region that does not contain an E2-binding site, even 
Figure 7. Brd4 recruits E2, which in turn prevents TFIID and pol II association with the HPV E6 promoter region. (A) E2 inhibits the expression of the E6 transcript. RT-PCR was performed using primer pairs annealing to the coding region of $\mathrm{E} 2, \mathrm{E} 6$, and $\beta$-actin, respectively. (B) Recruitment of E2 prevents TFIID and pol II association with the E6 promoter region. ChIP assays were conducted with chromatin samples isolated from HeLa and HeLa-E2 cells using different anti-protein antibodies or no antibody (mock) as indicated. PCR products, amplified by a primer pair annealing to the HPV-18 E6 promoter-proximal region (see $C$ ), were resolved on an $8 \%$ polyacrylamide gel and visualized after ethidiumbromide staining. $(C)$ Locations of HPV-18specific primer pairs used for ChIP assays. $(D)$ Treatment of HeLa-E2 cells with sodium butyrate enhances Brd4-mediated recruitment of E2 to the endogenous HPV-18 E6 promoter and the $-0.5-\mathrm{kb}$ region that contains an E2binding site. ChIP assays were performed with primer pairs outlined in C. $(E)$ Brd4 enhances E2 recruitment to the E6 promoter region. ChIP assay was performed with anti-FLAG M2 or anti-E2 antibodies with chromatin samples isolated from C-33A cells transfected with pGL7072-161, with (+) or without (-) E2, dsE2C, and f:Brd4 expression plasmids as indicated. PCR products, amplified by a primer pair annealing to the HPV-11 E6 promoter-proximal region (see the top drawing), were resolved on an $8 \%$ polyacrylamide gel and visualized after ethidium-bromide staining. $(F)$ The CTD and a bromodomain-containing region are both necessary for Brd4-mediated recruitment of E2 to the endogenous HPV-18 E6 promoter in stable HeLa-derived Brd4-knockdown cells (HeLa-shBrd4) cotransfected with HA:E2 and various Brd4 expression plasmids. The ChIP assay was performed with a primer pair that amplifies the HPV-18 E6 promoter region (see $C$ ).

though the level of acetylated chromatin at the $-1-\mathrm{kb}$ region was significantly enhanced by sodium butyrate treatment (Fig. 7D, cf. lanes 4 and 9, lanes 5 and 10, third row). This finding suggests that acetylated chromatin alone is not sufficient to stabilize Brd4 binding to chromatin and a synergistic binding between Brd4 and a sequence-specific DNA-binding protein that interacts with Brd4, such as E2, is necessary for mutual enhancement of binding to acetylated chromatin. This view is also consistent with the rapid "on" and "off" mode of Brd4 binding to acetylated chromatin in living cells (Dey et al. 2003). It should be mentioned that, although Brd4 is efficiently recruited to HPV chromatin, it was not universally found in every gene, such as the protocadherin y3A1 gene (S.-Y. Wu and C.-M. Chiang, unpubl.).

The recruitment of E2 by Brd4 was further examined by conducting a transient transfection-ChIP assay in sodium butyrate-treated C-33A cells to evaluate the efficiency of $\mathrm{E} 2$ and dsE2C recruitment to the E6 promoter in pGL7072-161, with or without f:Brd4. As shown in Figure 7E, f:Brd4 alone bound efficiently to the E6 promoter region, irrespective of the presence or absence of E2 and dsE2C (Fig. 7E, cf. lanes 2 and 4 and 6 in $\alpha$-FLAG row). In contrast, recruitment of E2 could be enhanced when exogenous f:Brd4 also associates with the E6 promoter region (Fig. 7E, lanes 3,4, bottom two rows). Not surprisingly, Brd4 did not influence the recruitment of dsE2C (Fig. 7E, lanes 5,6, $\alpha$-E2 row), since dsE2C lacks the $\mathrm{N}$-terminal domain needed for Brd4 interaction. The Brd4-independent recruitment of dsE2C to the E6 promoter is likely caused by overexpression of dsE2C (see Fig. 6A, lane 2), which has a tendency to bind nucleosome-free DNA templates or improperly assembled chromatin typically observed with transfected reporter plasmids.

To further define the role of Brd4 in E2 recruitment, we cotransfected an HA-tagged E2 expression plasmid with either the full-length or a truncated Brd4 expression construct into a stable HeLa-derived Brd4-knockdown cell line (HeLa-shBrd4) and monitored the recruitment of E2 and various Brd4 mutants (see Fig. 6D) to the endogenous HPV-18 E6 promoter region. As shown in Figure $7 F$, only full-length and the 280-1400 and 1-1260 mutants, but not the 600-1400 mutant, could be recruited to the E6 promoter, indicating that the presence of at least a bromodomain is necessary for Brd4 targeting to HPV chromatin (Fig. 7F, lanes 2-5, second row). A reduced recruitment of the BDI-deleted 280-1400 mutant to the E6 promoter, compared with the full-length Brd4 (Fig. 7F, lanes 2,3, second row), correlated with slightly diminished activity of 280-1400 in restoring E2-mediated repression in \#1-13 Brd4-knockdown cells (see Fig. 
6E, lanes 2 vs. 3). Deletion of the CTD (1-1260) or both BDI and BDII bromodomains (600-1400) severely impaired E2 recruitment to the E6 promoter (Fig. 7F, lanes 4,5 vs. 2,3), likely accounting for the deficiency of these two Brd4 mutants in supporting E2-mediated repression (see Fig. 6E, lanes 4,5). From these experiments, we conclude that the chromatin targeting activity of Brd4 and its E2-interacting CTD are both necessary for Brd4-mediated recruitment of E2 to HPV chromatin and for transcriptional silencing of the E6 promoter by preventing preinitiation complex assembly via blocking TFIID and pol II binding to the target gene.

\section{Discussion}

From the biochemical purification of a major HPV-11 E2-assembled cellular complex, we were able to identify by mass spectrometry the double bromodomain protein Brd4 as the predominant cellular protein naturally associated with E2 in human cells. Using in vitro reconstituted HPV chromatin that faithfully recapitulates in vivo nucleosome positioning on the $\mathrm{E} 6$ promoter region, we demonstrated that the isolated Brd4-E2 complex functions as a transcriptional silencer capable of inhibiting HPV chromatin transcription mediated by the human c-Jun/c-Fos dimeric AP-1 complex. This inhibition is sequence-dependent and occurs primarily through the \#4 promoter-proximal E2-binding site. Recombinant Brd4 and E2 are sufficient for the silencing activity, which requires both the $\mathrm{N}$-terminal region and the $\mathrm{C}$ terminal DNA-binding domain of E2 as well as the extreme $\mathrm{C}$ terminus and a bromodomain-containing region of Brd4. The ability of Brd4 and E2 to inhibit E6 promoter activity was further illustrated in vivo by reporter gene assays and RT-PCR using transfected and stable Brd4knockdown cells. By conducting ChIP assays to monitor recruitment of transcriptional components to the endogenous HPV-18 E6 promoter in HeLa and HeLa-derived E2-expressing cells and to a transiently transfected HPV11 URR-E6 promoter-driven reporter, we found that Brd4 enhances E2 recruitment to the E6 promoter, likely depending on Brd4 binding to acetylated histones $\mathrm{H} 3$ and H4. Promoter-bound E2 then suppresses transcription by preventing TFIID binding to the TATA box and also pol II entry to the E6 promoter region. These findings are significant in several aspects.

First, the identification of Brd4 as the cellular corepressor mediating E2 inhibition of E6 promoter activity through its interaction with the $\mathrm{N}$-terminal domain of E2 resolves a decade-old puzzle of why the $\mathrm{N}$-terminal region of $\mathrm{E} 2$, while dispensable for transcriptional repression in vitro with DNA templates, is necessary for E2mediated repression in vivo (see introduction above). The hypothesized chromatin-binding corepressor (Hou et al. 2000) is now identified as Brd4. Second, the identification of AP-1, but not Sp1 and YY1 (data not shown), as a critical cellular transcription factor able to initiate transcription in vitro from silenced HPV chromatin, whose structure was first observed nearly 30 years ago by electron microscopy with viral samples obtained from human plantar warts (Favre et al. 1977), directly demonstrates the involvement of AP-1 in HPV transcription and is consistent with genetic studies indicating the importance of AP-1-binding sites for E6/E7 gene expression in both differentiated and undifferentiated cell types, including keratinocytes and stratified epithelial tissues (Kyo et al. 1997; Zhao et al. 1997; and references therein). Third, the finding that acetylation on histones $\mathrm{H} 3$ and H4 remains unaltered following Brd4-mediated recruitment of E2 to the E6 promoter region indicates that acetylation on histone tails may also signal the recruitment of a sequence-specific transcriptional repressor whose chromatin targeting activity depends on the binding of a corepressor to acetylated chromatin. Fourth, the inhibition of TFIID and pol II association with the E6 promoter resulting from E2 binding in vivo is in agreement with previous in vitro studies showing that E2 is able to block TBP binding to the TATA box (Dostatni et al. 1991; Hou et al. 2000) and independently impedes TFIIF-escorted pol II entry to the E6 promoter region (Hou et al. 2000). Taken together, these functional studies and recruitment assays provide a molecular mechanism underlying the function of Brd4 in linking chromatin targeting to E2-mediated HPV transcriptional silencing.

Chromatin targeting by bromodomain-containing proteins has been commonly implicated in gene activation (see introduction above). The recent reports that Brd4 can function as a transcriptional coactivator capable of stimulating the kinase and elongation activities of $\mathrm{P}$ TEFb (Jang et al. 2005; Yang et al. 2005) provides the first evidence for Brd4's involvement in transcription, in addition to its previously characterized roles in cell proliferation (Dey et al. 2000; Houzelstein et al. 2002), DNA replication (Maruyama et al. 2002), and gene rearrangement found in $\mathrm{t}(15 ; 19)$-associated carcinomas (French et al. 2003). Our finding that Brd4 can also act as a transcriptional corepressor implicated in HPV gene silencing not only extends the functional properties of Brd4 but also exemplifies a dual role of a typical transcription cofactor in gene activation and repression (Thomas and Chiang 2006). In our purified E2-P.5 complex, we could not detect the cyclin $\mathrm{T} 1$ and $\mathrm{Cdk} 9$ components of $\mathrm{P}$ $\mathrm{TEFb}$, nor the presence of Brd2 (S.-Y. Wu and C.-M. Chiang, unpubl.). This is not surprising, given that $\mathrm{P}$ TEFb-Brd4 interaction seems rather weak and tends to be disrupted by salt concentrations $>0.25 \mathrm{M} \mathrm{KCl}$ (Yang et al. 2005) and that Brd2 cannot replace Brd4 in supporting E2-mediated repression in Brd4-knockdown cells (S.-Y. $\mathrm{Wu}$ and C.-M. Chiang, unpubl.).

The finding that Brd4 is an HPV-11 E2 corepressor is also intriguing from the virus perspective. Brd4 has recently been identified as the cellular adaptor mediating bovine papillomavirus type 1 (BPV-1) genome tethering to host mitotic chromosomes through its interaction with BPV-1 E2, thereby facilitating viral genome segregation during mitosis (You et al. 2004). This genometethering function of BPV-1 E2 (Lehman and Botchan 1998; Skiadopoulos and McBride 1998; Ilves et al. 1999) can be adapted for plasmid maintenance in Saccharomy- 
ces cerevisiae, provided that E2-binding sites are created in the plasmid and mammalian Brd4 is additionally introduced into yeast (Brannon et al. 2005). The association between Brd4 and BPV-1 E2 with chromatin appears to be stable and persists through both mitosis and interphase (McPhillips et al. 2005). Since HPV E2 does not stably associate with mitotic chromosomes and seems to anchor on mitotic spindles during mitosis /Van Tine et al. 2004), it remains to be investigated whether the genomic-tethering function of BPV-1 E2 can be applied to HPV E2 with respect to their functional interactions with Brd4. Likewise, it needs to be defined whether the transcriptional silencing activity of the HPV E2-Brd4 complex can also be observed with E2 complexes isolated from animal papillomaviruses. Undoubtedly, Brd4 is a central player in virus-induced pathogenesis and is evidently an appealing molecular target for future drug development to control HPV-induced human diseases, including genital warts, skin tumors, and cervical cancers.

\section{Materials and methods}

\section{Plasmid construction}

Retroviral expression plasmids pBPSTR-6HisF:11E2 and pBPSTR-6HisF:CM4 were used to generate HeLa- and 293-derived cell lines that conditionally express hexahistidine/FLAGtagged HPV-11 E2 and dsE2C, respectively. Baculovirus transfer plasmids pVL-F:mBrd4 and pVL-HA:E2 were used individually for expression and purification of FLAG-tagged mouse Brd4 and HA-tagged HPV-11 E2 in insect Sf9 cells. Construction of these plasmids as well as the other bacterial and mammalian expression plasmids for E2 and Brd4 is described in the Supplemental Material.

Two retroviral plasmids, phBrd4-shRNA\#1 and phBrd4shRNA\#2, which contain human Brd4 nucleotide sequences 228-246 and 423-441 (relative to the translational start site), were constructed, respectively, by cloning a 59-mer doublestranded oligonucleotide, annealed from 5'-GATCCCC-19 nt (sense)-TTCAAGAGA-19 nt (antisense)-TTTTT-3' and 5'AGCTAAAAA-19 nt-TCTCTTGAA-19 nt-GGG-3' that harbor the individual Brd4 targeting sequence (i.e., 228-246 or 423441), into pSUPERIOR.retro.neo+gfp (OligoEngine) between BglII and HindIII sites.

\section{Establishment of inducible E2 cell lines and stable Brd4-knockdown cells}

Tetracycline-regulated 293-E2, 293-dsE2C, HeLa-E2, and HeLadsE2C cell lines that conditionally express hexahistidine/ FLAG-tagged E2 and hexahistidine/FLAG-tagged dsE2C in 293 or $\mathrm{HeLa}$ cells were established by transfecting pBPSTR6HisF:11E2 and pBPSTR-6HisF:CM4 into the $\Psi_{\text {CRIP }}$ retroviruspackaging cell line, from which the resulting viruses were used to infect human 293 or HeLa S cells according to the published protocols (Wu and Chiang 2001b; Wu et al. 2003). After selection of infected cells with $0.5 \mu \mathrm{g} / \mathrm{mL}$ puromycin in DMEM containing $10 \%$ fetal bovine serum and $1 \mu \mathrm{g} / \mathrm{mL}$ tetracycline for $\sim 3$ wk, antibiotic-resistant colonies were individually expanded into cell lines and tested for inducible expression of hexahistidine/FLAG-tagged E2 or hexahistidine/FLAG-tagged dsE2C by Western blotting with anti-hexahistidine antibodies.

The stable C-33A-derived Brd4-knockdown cell line \#1-13 and the vector-harboring cell line V-1 were established by transfecting phBrd4-shRNA\#1 and pSUPERIOR.retro.neo+gfp into $\Psi_{\text {CRIP }}$ cells, in which the resulting viruses were used to infect C-33A cells, following the same protocols described above, except $1 \mathrm{mg} / \mathrm{mL}$ G418 was used for selecting drug-resistant colonies in the absence of tetracycline. The stable HeLa-derived shBrd4-knockdown cells (\#1-3) were similarly established in HeLa S cells as described above.

\section{Purification of the E2-P.5 complex and identification of Brd4}

The E2-P.5 complex was purified by immunoaffinity purification and peptide elution methods (Wu et al. 1998) from the 0.5 $\mathrm{M} \mathrm{KCl}$ fraction of the P11 ion-exchange column (P.5) after fractionation of 293-E2 nuclear extracts as described (Kershnar et al. 1998). Control purification was similarly conducted using the P.5 fraction derived from nuclear extracts prepared from 293 and 293-dsE2C, respectively. For protein identification, see the Supplemental Material.

\section{Purification of recombinant proteins}

Purification of FLAG-tagged mouse Brd4, FLAG-tagged HPV-11 E2, in vivo assembled recombinant Brd4-E2 complexes, HeLa core histones, recombinant human NAP-1, p300, AP-1, Drosophila ACF, Gal4-VP16, and various FLAG-tagged mouse Brd4 deletion domains was conducted as described (Chiang and Roeder 1993; Wu and Chiang 1998; Wu et al. 1999) and is further detailed in the Supplemental Material.

\section{Chromatin assembly and in vitro transcription}

Chromatin assembly and in vitro transcription were performed as described (Thomas and Chiang 2005) with some modifications. Unless otherwise specified, relative transcription (Rel Txn) in each set of reactions is defined as the signal intensity, quantified by Typhoon 9200 PhosphorImager (GE Healthcare), from the HPV chromatin template relative to that performed in the presence of AP-1, acetyl-CoA, and p300, after initial normalization with the signal derived from the internal pML $\Delta 53$ control template. For specific details, see the Supplemental Material.

\section{Mapping nucleosome position by LM-PCR and primer extension}

In vivo nucleosome positioning on the HPV-18 E6 promoterproximal region was determined by LM-PCR using MNase-digested HeLa chromatin according to published protocols (Kemper et al. 2004). Specifically, MNase-digested HeLa chromatin was 5 '-phosphorylated by T4 DNA kinase and then ligated with a 23-mer DNA linker, before it was used for PCR amplification with an upstream linker primer and a downstream HPV-18 E6 promoter-specific primer that anneals to nucleotides 149-172 (for primer A mapping) or 7751-7777 (for primer B mapping) of the HPV-18 genome. Asymmetric PCR (or primer extension) was further conducted using PCR-amplified DNA fragments with ${ }^{32} \mathrm{P}$-labeled primer A that binds to nucleotides 111-136, or primer B hybridizing to nucleotides $91-118$ of the HPV-18 genome. The labeled products were separated on a $5 \%$ DNA sequencing gel and analyzed by the Typhoon 9200 PhosphorImager.

In vitro mapping of nucleosome position was conducted with in vitro reconstituted HPV-11 (p7072-70GLess/ $\mathrm{I}^{+}$) or HPV-18 (pBR322-HPV18) chromatin, which was treated with MNase for a different time period and then used for asymmetric PCR, after deproteinization, with ${ }^{32} \mathrm{P}$-labeled Gless(AS)-31/55 primer $\left(5^{\prime}\right.$ - 
AGGAATAATGAGGAAAGGAGAGTAG-3') for HPV-11 or ${ }^{32} \mathrm{P}$-labeled primer A for HPV-18. The products were analyzed as described above.

\section{GST pull-down assay}

For GST pull-down assays, $200 \mathrm{ng}$ of recombinant wild-type FLAG-tagged mouse Brd4 or $60 \mathrm{ng}$ of its deletion domains was incubated individually with immobilized GST-tagged fulllength or amino acids 1-200 of E2, or dsE2C, and processed as described (Wu and Chiang 2001a). The amounts of immobilized E2 proteins and bound Brd4 were analyzed by Western blotting with antibodies against GST and the FLAG epitope, respectively.

Luciferase reporter gene assay, RT-PCR, protein detection, and ChIP assays performed with cultured cells

See the Supplemental Material.

\section{Acknowledgments}

We thank G.V. Denis for anti-Brd2 antibodies, J.A. Goodrich for pET-6His-c-Fos and pET-Jun plasmids, P.M. Howley for the pBR322-HPV18 clone and pcDNA4c-hBrd4, and K. Ozato for the pBSBrd4 plasmid and anti-mouse Brd4 antibodies. We are also grateful to Alison S. Chiang for help in plasmid constructions, to Joanne Kim for help with mass spectrometry, and to P. Kaur, D. Samols, and M.C. Thomas for comments on the manuscript. This work was supported by RO1 grants CA103867 (to C.-M.C.) and DK062777 (to J.K.K.) from the National Institutes of Health, by NCI Cancer Center Support Grant P30 CA08748 (to P.T.), and Aging-Cancer Research Development Program P20 CA10373 (to Case Western Reserve University).

\section{References}

Abbate, E.A., Berger, J.M., and Botchan, M.R. 2004. The X-ray structure of the papillomavirus helicase in complex with its molecular matchmaker E2. Genes \& Dev. 18: 1981-1996.

Abroi, A., Ilves, I., Kivi, S., and Ustav, M. 2004. Analysis of chromatin attachment and partitioning functions of bovine papillomavirus type 1 E2 protein. J. Virol. 78: 2100-2113.

Bellanger, S., Demeret, C., Goyat, S., and Thierry, F. 2001. Stability of the human papillomavirus type $18 \mathrm{E} 2$ protein is regulated by a proteasome degradation pathway through its amino-terminal transactivation domain. J. Virol. 75: 72447251.

Bernard, B.A., Bailly, C., Lenoir, M.-C., Darmon, M., Thierry, F., and Yaniv, M. 1989. The human papillomavirus type 18 (HPV18) E2 gene product is a repressor of the HPV18 regulatory region in human keratinocytes. J. Virol. 63: 4317-4324.

Blachon, S., Bellanger, S., Demeret, C., and Thierry, F. 2005. Nucleo-cytoplasmic shuttling of high risk human papillomavirus E2 proteins induces apoptosis. J. Biol. Chem. 280: 36088-36098.

Botchan, M. 2004. Hitchhiking without covalent integration. Cell 117: 280-281.

Brannon, A.R., Maresca, J.A., Boeke, J.D., Basrai, M.A., and McBride, A.A. 2005. Reconstitution of papillomavirus E2-mediated plasmid maintenance in Saccharomyces cerevisiae by the Brd4 bromodomain protein. Proc. Natl. Acad. Sci. 102: 2998-3003.

Chiang, C.-M. and Roeder, R.G. 1993. Expression and purification of general transcription factors by FLAG epitope-tagging and peptide elution. Pept. Res. 6: 62-64.
Chiang, C.-M., Broker, T.R., and Chow, L.T. 1991. An E1M^ E2C fusion protein encoded by human papillomavirus type 11 is a sequence-specific transcription repressor. J. Virol. 65: 3317-3329.

Chiang, C.-M., Dong, G., Broker, T.R., and Chow, L.T. 1992. Control of human papillomavirus type 11 origin of replication by the $\mathrm{E} 2$ family of transcription regulatory proteins. J. Virol. 66: 5224-5231.

Demeret, C., Desaintes, C., Yaniv, M., and Thierry, F. 1997. Different mechanisms contribute to the E2-mediated transcriptional repression of human papillomavirus type 18 viral oncogenes. J. Virol. 71: 9343-9349.

Denis, G.V., Vaziri, C., Guo, N., and Faller, D.V. 2000. RING3 kinase transactivates promoters of cell cycle regulatory genes through E2F. Cell Growth Differ. 11: 417-424.

Dey, A., Ellenberg, J., Farina, A., Coleman, A.E., Maruyama, T., Sciortino, S., Lippincott-Schwartz, J., and Ozato, K. 2000. A bromodomain protein, MCAP, associates with mitotic chromosomes and affects $\mathrm{G}_{2}$-to-M transition. Mol. Cell. Biol. 20: 6537-6549.

Dey, A., Chitsaz, F., Abbasi, A., Miteli, T., and Ozato, K. 2003. The double bromodomain protein Brd4 binds to acetylated chromatin during interphase and mitosis. Proc. Natl. Acad. Sci. 100: 8758-8763.

Dostatni, N., Lambert, P.F., Sousa, R., Ham, J., Howley, P.M., and Yaniv, M. 1991. The functional BPV-1 E2 trans-activating protein can act as a repressor by preventing formation of the initiation complex. Genes \& Dev. 5: 1657-1671.

Dowhanick, J.J., McBride, A.A., and Howley, P.M. 1995. Suppression of cellular proliferation by the papillomavirus E2 protein. J. Virol. 69: 7791-7799.

Favre, M., Breitburd, F., Croissant, O., and Orth, G. 1977. Chromatin-like structures obtained after alkaline disruption of bovine and human papillomaviruses. J. Virol. 21: 1205-1209.

French, C.A., Miyoshi, I., Kubonishi, I., Grier, H.E., PerezAtayde, A.R., and Fletcher, J.A. 2003. BRD4-NUT fusion oncogene: A novel mechanism in aggressive carcinoma. Cancer Res. 63: 304-307.

Goodwin, E.C. and DiMaio, D. 2001. Induced senescence in HeLa cervical carcinoma cells containing elevated telomerase activity and extended telomeres. Cell Growth Differ. 12: 525-534.

Goodwin, E.C., Naeger, L.K., Breiding, D.E., Androphy, E.J., and DiMaio, D. 1998. Transactivation-competent bovine papillomavirus E2 protein is specifically required for efficient repression of human papillomavirus oncogene expression and for acute growth inhibition of cervical carcinoma cell lines. J. Virol. 72: 3925-3934.

Hegde, R.S. 2002. The papillomavirus E2 proteins: Structure, function, and biology. Annu. Rev. Biophys. Biomol. Struct. 31: 343-360.

Hou, S.Y., Wu, S.-Y., Zhou, T., Thomas, M.C., and Chiang, C.-M. 2000. Alleviation of human papillomavirus E2-mediated transcriptional repression via formation of a TATA binding protein (or TFIID)-TFIIB-RNA Polymerase II-TFIIF preinitiation complex. Mol. Cell. Biol. 20: 113-125.

Hou, S.Y., Wu, S.-Y., and Chiang, C.-M. 2002. Transcriptional activity among high and low risk human papillomavirus E2 proteins correlates with E2 DNA binding. J. Biol. Chem. 277: 45619-45629.

Houzelstein, D., Bullock, S.L., Lynch, D.E., Grigorieva, E.F., Wilson, V.A., and Beddington, R.S.P. 2002. Growth and early postimplantation defects in mice deficient for the bromodomain-containing protein Brd4. Mol. Cell. Biol. 22: 37943802.

Hwang, E.-S., Riese II, D.J., Settleman, J., Nilson, L.A., Honig, J., 
Flynn, S., and DiMaio, D. 1993. Inhibition of cervical carcinoma cell line proliferation by the introduction of a bovine papillomavirus regulatory gene. J. Virol. 67: 3720-3729.

Ilves, I., Kivi, S., and Ustav, M. 1999. Long-term episomal maintenance of bovine papillomavirus type 1 plasmids is determined by attachment to host chromosomes, which is mediated by the viral E2 protein and its binding sites. J. Virol. 73: 4404-4412.

Jacobson, R.H., Ladurner, A.G., King, D.S., and Tjian, R. 2000. Structure and function of a human $\mathrm{TAF}_{\mathrm{II}} 250$ double bromodomain module. Science 288: 1422-1425.

Jang, M.K., Mochizuki, K., Zhou, M., Jeong, H.-S., Brady, J.N., and Ozato, K. 2005. The bromodomain protein $\mathrm{Brd} 4$ is a positive regulatory component of P-TEFb and stimulates RNA polymerase II-dependent transcription. Mol. Cell 19: 523-534.

Jeckel, S., Loetzsch, E., Huber, E., Stubenrauch, F., and Iftner, T. 2003. Identification of the E9^E2C cDNA and functional characterization of the gene product reveal a new repressor of transcription and replication in cottontail rabbit papillomavirus. J. Virol. 77: 8736-8744.

Kanno, T., Kanno, Y., Siegel, R.M., Jang, M.K., Lenardo, M.J., and Ozato, K. 2004. Selective recognition of acetylated histones by bromodomain proteins visualized in living cells. Mol. Cell 13: 33-43.

Kemper, J.K., Kim, H., Miao, J., Bhalla, S., and Bae, Y. 2004. Role of an mSin3A-Swi/Snf chromatin remodeling complex in the feedback repression of bile acid biosynthesis by SHP. Mol. Cell. Biol. 24: 7707-7719.

Kershnar, E., Wu, S.-Y., and Chiang, C.-M. 1998. Immunoaffinity purification and functional characterization of human transcription factor IIH and RNA polymerase II from clonal cell lines that conditionally express epitope-tagged subunits of the multiprotein complexes. J. Biol. Chem. 273: 34444 34453.

Kyo, S., Klumpp, D.J., Inoue, M., Kanaya, T., and Laimins, L.A. 1997. Expression of AP1 during cellular differentiation determines human papillomavirus E6/E7 expression in stratified epithelial cells. J. Gen. Virol. 78: 401-411.

Lehman, C.W. and Botchan, M.R. 1998. Segregation of viral plasmids depends on tethering to chromosomes and is regulated by phosphorylation. Proc. Natl. Acad. Sci. 95: 43384343.

Maruyama, T., Farina, A., Dey, A., Cheong, J., Bermudez, V.P., Tamura, T., Sciortino, S., Shuman, J., Hurwitz, J., and Ozato, K. 2002. A mammalian bromodomain protein, brd4, interacts with replication factor $\mathrm{C}$ and inhibits progression to $\mathrm{S}$ phase. Mol. Cell. Biol. 22: 6509-6520.

Matangkasombut, O., Buratowski, R.M., Swilling, N.W., and Buratowski, S. 2000. Bromodomain factor 1 corresponds to a missing piece of yeast TFIID. Genes \& Dev. 14: 951-962.

McBride, A.A., McPhillips, M.G., and Oliveira, J.G. 2004. Brd4: Tethering, segregation and beyond. Trends Microbiol. 12: 527-529.

McPhillips, M.G., Ozato, K., and McBride, A.A. 2005. Interaction of bovine papillomavirus E2 protein with Brd4 stabilizes its association with chromatin. J. Virol. 79: 8920-8932.

Mellor, J. 2005. The dynamics of chromatin remodeling at promoters. Mol. Cell 19: 147-157.

Münger, K., Baldwin, A., Edwards, K.M., Hayakawa, H., Nguyen, C.L., Owens, M., Grace, M., and Huh, K. 2004 Mechanisms of human papillomavirus-induced oncogenesis. J. Virol. 78: 11451-11460.

Skiadopoulos, M.H. and McBride, A.A. 1998. Bovine papillomavirus type 1 genomes and the E2 transactivator protein are closely associated with mitotic chromatin. J. Virol. 72: 2079-
2088.

Stenlund, A. 2003. Initiation of DNA replication: Lessons from viral initiator proteins. Nat. Rev. Mol. Cell Biol. 4: 777-785.

Stubenrauch, F., Hummel, M., Iftner, T., and Laimins, L.A. 2000. The E8 ${ }^{\wedge} E 2 C$ protein, a negative regulator of viral transcription and replication, is required for extrachromosomal maintenance of human papillomavirus type 31 in keratinocytes. J. Virol. 74: 1178-1186.

Stünkel, W. and Bernard, H.-U. 1999. The chromatin structure of the long control region of human papillomavirus type 16 represses viral oncoprotein expression. J. Virol. 73: 19181930.

Thomas, M.C. and Chiang, C.-M. 2005. E6 oncoprotein represses p53-dependent gene activation via inhibition of protein acetylation independently of inducing p53 degradation. Mol. Cell 17: 251-264.

. 2006. The general transcription machinery and general cofactors. Crit. Rev. Biochem. Mol. Biol. 41: 105-178.

Van Tine, B.A., Dao, L.D., Wu, S.-Y., Sonbuchner, T.M., Lin, B.Y., Zou, N., Chiang, C.-M., Broker, T.R., and Chow, L.T. 2004. Human papillomavirus (HPV) origin-binding protein associates with mitotic spindles to enable viral DNA partitioning. Proc. Natl. Acad. Sci. 101: 4030-4035.

Winston, F. and Allis, C.D. 1999. The bromodomain: A chromatin-targeting module? Nat. Struct. Biol. 6: 601-604.

Wu, S.-Y. and Chiang, C.-M. 1998. Properties of PC4 and RNA polymerase II complex in directing activated and basal transcription in vitro. J. Biol. Chem. 273: 12492-12498.

2001a. TATA-binding protein-associated factors enhance the recruitment of RNA polymerase II by transcriptional activators. J. Biol. Chem. 276: 34235-34243.

- 2001b. Expression and purification of epitope-tagged multisubunit protein complexes from mammalian cells. In Current protocols in molecular biology (eds. F.M. Ausubel et al.), pp. 16.22.1-16.22.17. Wiley, New York.

Wu, S.-Y., Kershnar, E., and Chiang, C.-M. 1998. TAF II $^{-i n d e p e n-~}$ dent activation mediated by human TBP in the presence of the positive cofactor PC4. EMBO J. 17: 4478-4490.

Wu, S.-Y., Thomas, M.C., Hou, S.Y., Likhite, V., and Chiang, C.-M. 1999. Isolation of mouse TFIID and functional characterization of TBP and TFIID in mediating estrogen receptor and chromatin transcription. J. Biol. Chem. 274: 2348023490

Wu, S.-Y., Zhou, T., and Chiang, C.-M. 2003. Human Mediator enhances activator-facilitated recruitment of RNA polymerase II and promoter recognition by TATA-binding protein (TBP) independently of TBP-associated factors. Mol. Cell. Biol. 23: 6229-6242.

Yang, Z., Yik, J.H.N., Chen, R., He, N., Jang, M.K., Ozato, K., and Zhou, Q. 2005. Recruitment of P-TEFb for stimulation of transcriptional elongation by the bromodomain protein Brd4. Mol. Cell 19: 535-545.

You, J., Croyle, J.L., Nishimura, A., Ozato, K., and Howley, P.M. 2004. Interaction of the bovine papillomavirus E2 protein with Brd4 tethers the viral DNA to host mitotic chromosomes. Cell 117: 349-360.

Zhao, W., Chow, L.T., and Broker, T.R. 1997. Transcription activities of human papillomavirus type 11 E6 promoterproximal elements in raft and submerged cultures of foreskin keratinocytes. J. Virol. 71: 8832-8840. 


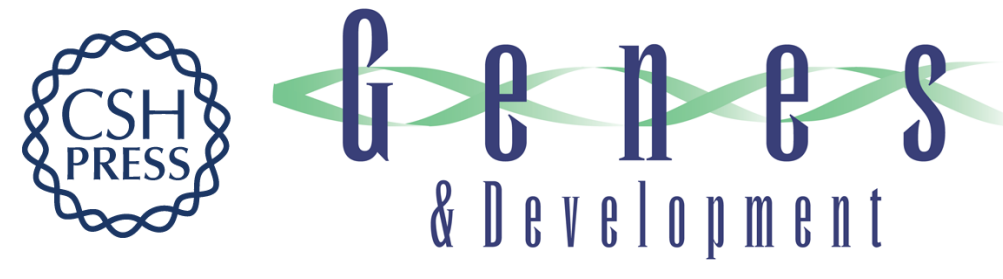

\section{Brd4 links chromatin targeting to HPV transcriptional silencing}

Shwu-Yuan Wu, A-Young Lee, Samuel Y. Hou, et al.

Genes Dev. 2006, 20:

Access the most recent version at doi:10.1101/gad.1448206

Supplemental

Material

References

License

Email Alerting Service
http://genesdev.cshlp.org/content/suppl/2006/09/05/gad.1448206.DC1

This article cites 56 articles, 42 of which can be accessed free at: http://genesdev.cshlp.org/content/20/17/2383.full.html\#ref-list-1

Receive free email alerts when new articles cite this article - sign up in the box at the top right corner of the article or click here.

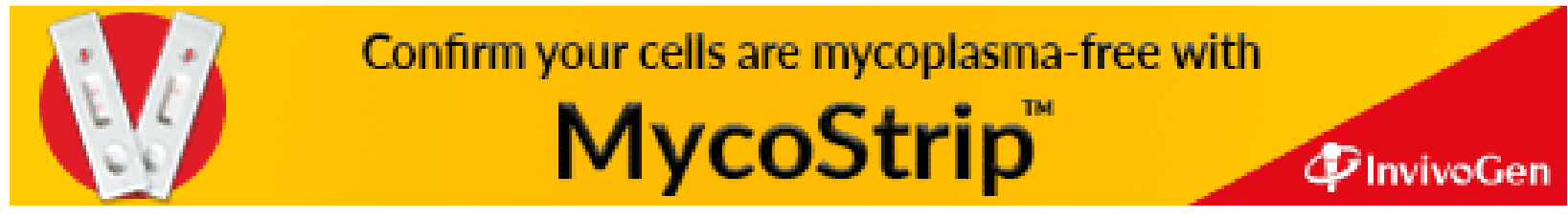

